\title{
The necklace Lie coalgebra and renormalization algebras
}

\author{
Wee Liang Gan and Travis Schedler
}

\begin{abstract}
We give a natural monomorphism from the necklace Lie coalgebra, defined for any quiver, to Connes and Kreimer's Lie coalgebra of trees, and extend this to a map from a certain quiver-theoretic Hopf algebra to Connes and Kreimer's renormalization Hopf algebra as well as to pre-Lie versions. These results are direct analogues of Turaev's results in 2004, by replacing algebras of loops on surfaces with algebras of paths on quivers. We also factor the morphism through an algebra of chord diagrams and explain the geometric version. We then describe how all of the Hopf algebras are uniquely determined by the pre-Lie structures and discuss noncommutative versions of the Hopf algebras.
\end{abstract}

Mathematics Subject Classification (2000). 17B99, 16W30.

Keywords. Necklace, renormalization, Connes-Kreimer, pre-Lie, tree-structure.

\section{Introduction}

Motivated by an attempt to understand the moduli space of flat connections on a vector bundle over a surface, Goldman constructed in [Gol84] a Lie bracket on the free vector space spanned by homotopy classes of (basepoint-free) loops on a surface, together with a Lie homomorphism (by taking trace of holonomy) to the Poisson algebra of functions on the aforementioned moduli space.

In [Tur91], Turaev discovered that one may define, in a similar way, a cobracket on the aforementioned space of loops, which is compatible with Goldman's bracket and yields a Lie bialgebra. He also constructed a quantization of this Lie bialgebra in terms of link diagrams on the surface.

This left open the question to find an interpretation of the Lie coalgebra. In [Tur05], Turaev discovered a relation between his Lie coalgebra and Connes and Kreimer's renormalization algebras [Kre98], [CK98], which form part of the algebraic foundations of perturbative quantum field theory. Specifically, he found a homomorphism from an up-to-isotopy, pointed version of his Lie coalgebra of loops to Connes and Kreimer's Lie coalgebra of trees, and constructed from this a commutative Hopf 
algebra on the loop side ${ }^{1}$ mapping to Connes and Kreimer's renormalization Hopf algebra. This allows one to interpret the combinatorics of loops and Turaev's Lie coalgebra as Feynman diagrams connected to renormalization.

Turaev found that additional structure from the construction is preserved, and accordingly generalized Connes and Kreimer's algebras to include the extra data. This attached to the trees the following: (1) a homotopy class of loops assigned to each vertex; (2) orientations on the edges; and (3) a ribbon graph structure.

An essential step of the above construction is the observation that, when one has a basepoint, Turaev's Lie coalgebra actually arises from a more fundamental pre-Lie coalgebra structure. In the basepoint-free case, Turaev constructed an "oriented trees" version of Connes and Kreimer's Lie coalgebra (which does not come from a pre-Lie coalgebra), so that one is still equipped with a homomorphism.

There is a known analogue for quivers of the Goldman/Turaev Lie bialgebra of loops, called the necklace Lie bialgebra. Here the Lie algebra was discovered for much the same reason as the Goldman algebra: because of its representation into the Poisson algebra of functions on the corresponding quiver variety [Gin01] (the Lie algebra was independently discovered in [BLB02]). In [Sch05], the second author constructed the cobracket and quantized the resulting Lie bialgebra, following in analogy with [Tur91]. It is thus natural to ask for an interpretation of the cobracket in terms of representations and, in particular, if all of the above results from [Tur05] can be generalized to the quiver setting.

In this note we answer this question affirmatively and present quiver analogues of the results of [Tur05]. We define a pre-Lie coalgebra on the quiver side in the rooted (basepointed) case. We also define a commutative "renormalization" Hopf algebra associated to any quiver. ${ }^{2}$ Then our main theorem (Theorem 3.3.16) is the construction of a natural monomorphism from these quiver algebras and the (oriented) necklace Lie coalgebra to the Connes and Kreimer algebras, with additional quivertheoretic structure we define. We explain how the pre-Lie and Hopf algebra structures are essentially equivalent, in all cases (necklaces, trees (Connes-Kreimer) and loops (Turaev)), using a general result about pre-Lie algebras [OG05].

Unlike in Turaev's case, for quivers there is no distinction between "up to isotopy" and "up to homotopy," so there is only one Lie coalgebra to consider in each of the oriented/rooted cases, which is the one that is compatible with the necklace bracket in the oriented case. In contrast, the isotopy coalgebra from [Tur05] is not compatible with the Goldman Lie bracket (compatibility requires passing to homotopy classes).

For quivers, we find that introducing a basepoint is the same as cutting a necklace

\footnotetext{
${ }^{1}$ This Hopf algebra differs significantly from the Hopf algebra mentioned in the previous paragraph: aside from the previous one referring to homotopy classes rather than isotopy classes, the Hopf algebra here is commutative, unlike the one of the previous paragraph.

${ }^{2}$ As in Turaev's case, this Hopf algebra is quite different from the quantized necklace algebra of [Sch05]: the latter is noncommutative, unlike the former; and the former involves paths which are not mod commutators, unlike the latter.
} 
and considering algebras of paths, so our algebras in the rooted case are actually algebras of paths in the quiver. The additional structure attached to trees in our setting replaces the loops (with basepoint) attached to vertices with cyclic paths (paths) in the quiver. The rest of the structure - the ribbon graph structure and orientation of edges - is unchanged.

Furthermore, we discover that the monomorphisms factor through a "chord algebra" we define, which essentially is the span of necklaces with certain chord diagrams (Theorem 3.4.10). This clarifies the construction and the reason that it exists. We also briefly describe the corresponding geometric object, in the setting of [Tur05], where the chord diagrams become geometric chord diagrams [AMR96] (chord algebras were not mentioned in [Tur05]).

Finally, we briefly give a noncommutative version of the Hopf algebra analogous to Section 8.5 of [Tur05], which maps to Foissy's noncommutative algebra of labeled rooted trees [Foi02]. In particular, this shows that paths and loops have a noncommutative (= ordering) structure which does not exist for rooted trees without labels. The noncommutative Hopf algebras, unlike their commutative counterparts, are not determined by the pre-Lie structure alone (which essentially forgets about the "labelings" on the associated trees).

The organization of the paper is as follows. In Section 2, we briefly recall the necessary definitions from [Kre98], [CK98], [Tur05] (for the tree side), and from [Sch05] (for the quiver side). Then, in Section 3, we define the new quiver-theoretic Hopf algebra and pre-Lie coalgebra, state our main result, and generalize it through chord algebras. We also explain the equivalence of pre-Lie coalgebras and commutative Hopf algebras of a special form, and give the noncommutative version of the constructions in this paper. Finally, in Section 4, we provide the postponed proofs (e.g., of the main theorem).

Acknowledgements. We are grateful to Victor Ginzburg for connecting the authors and for some useful comments. We thank Muriel Livernet for helpful comments and references. The first author was partially supported by NSF grant DMS-0726154. The second author was partially supported by an NSF GRF.

\subsection{Notation}

Notation 1.1.1. Throughout, $\mathbb{k}$ denotes a fixed commutative ring with unit.

Notation 1.1.2. For any permutation $\sigma \in \Sigma_{n}$, we define $\tau_{\sigma}: V_{1} \otimes V_{2} \otimes \cdots \otimes V_{n} \rightarrow$ $V_{\sigma^{-1}(1)} \otimes V_{\sigma^{-1}(2)} \otimes \cdots \otimes V_{\sigma^{-1}(n)}$ as the permutation of components corresponding to $\sigma$.

Notation 1.1.3. We use cycle notation for our permutations. Thus, (123) denotes the permutation $1 \mapsto 2 \mapsto 3 \mapsto 1$. 


\section{Tree algebras}

In this section we recall the needed constructions of [Tur05], [Kre98], [CK98].

2.1. Algebras of trees. We recall the Lie coalgebra and Hopf algebra of rooted trees from [Kre98], [CK98], following [Tur05], where the former is generalized to a pre-Lie coalgebra and to the setting of oriented trees.

2.1.1. Pre-Lie (co)algebras. We recall first the definition of pre-Lie algebras (independently discovered by [Ger63] and [Vin63]) and their dual, pre-Lie coalgebras (following [Tur05]). Note that there is a wide variety of important pre-Lie algebras, including the Hochschild cochain complex of an algebra, vector fields on affine space, and the examples in this paper.

A (left) pre-Lie algebra over $\mathbb{k}$ is a $\mathbb{k}$-module with a $\mathbb{k}$-bilinear product $\star$ satisfying

$$
(x \star y) \star z-x \star(y \star z)=(y \star x) \star z-y \star(x \star z) .
$$

If $\star$ is a pre-Lie multiplication, then $[x, y]:=x \star y-y \star x$ must be a Lie bracket.

To obtain the definition of pre-Lie coalgebra, we dualize in the sense of determining what structure exists on $\mathfrak{g}^{*}$ if $\mathfrak{g}$ is a pre-Lie algebra. Precisely, a (left) pre-Lie coalgebra, $\mathfrak{g}$, over $\mathbb{k}$ is a $\mathbb{k}$-module with a $\mathbb{k}$-linear map $\delta_{0}: \mathfrak{g} \rightarrow \mathfrak{g} \otimes \mathfrak{g}$ satisfying

$$
\left(\operatorname{Id}-\tau_{(12)}\right)\left(\delta_{0} \otimes 1-1 \otimes \delta_{0}\right) \delta_{0}=0 \in \operatorname{Hom}_{\mathfrak{k}}(\mathfrak{g}, \mathfrak{g} \otimes \mathfrak{g} \otimes \mathfrak{g}) .
$$

If $\delta_{0}$ is a pre-Lie comultiplication, then $\delta:=\delta_{0}-\tau_{(12)} \delta_{0}$ must be a Lie cobracket.

2.1.2. The pre-Lie coalgebra of rooted trees and Lie coalgebra of oriented trees. A rooted tree $T$ is a collection of vertices $V(T)$ and edges $E(T)$, and a map $E(T) \rightarrow$ $V(T)^{(2)}$ from edges to unordered pairs of vertices such that the resulting graph is connected and has no cycles (or loops), together with a distinguished vertex, called the root.

An oriented tree is the same but without the distinguished vertex, and whose edge map is actually a map $E(T) \rightarrow V(T)^{2}$ from edges to ordered pairs of vertices.

Let $\widetilde{\mathcal{T}}_{\mathrm{rt}}$ be the free $\mathrm{k}$-module with basis given by the isomorphism classes of rooted trees. In [CK98] ([Tur05] for the "pre-"), the following pre-Lie comultiplication $\delta_{\mathrm{p}, \mathrm{rt}}$ is defined on $\mathcal{T}_{\mathrm{rt}}$ :

$$
\delta_{\mathrm{p}, \mathrm{rt}}(T)=\sum_{e \in E(T)} T_{e}^{1} \otimes T_{e}^{2},
$$

where $T_{e}^{1}, T_{e}^{2}$ are the trees obtained by deleting the edge $e$ and $T_{e}^{2}$ is the tree which contains the root. The root of $T_{e}^{1}$ is the vertex which was incident to $e$, and the root of $T_{e}^{2}$ is the root of $T$.

Proposition 2.1.2 ([Tur05]). The map $\delta_{\mathrm{p}, \mathrm{rt}}$ is a pre-Lie comultiplication. 
Thus, one deduces that the skew-symmetrization $\delta_{\mathrm{rt}}:=\delta_{\mathrm{p}, \mathrm{rt}}-\tau_{(12)} \delta_{\mathrm{p}, \mathrm{rt}}$ is a Lie cobracket, which was already discovered in [CK98] (and motivated the above result).

In [Tur05], a version for oriented trees is also given. Let $\mathcal{T}_{\text {or }}$ be the free $\mathbb{k}$-module with basis given by the isomorphism classes of oriented trees. Then a Lie cobracket $\delta_{\text {or }}: \mathcal{T}_{\text {or }} \rightarrow \mathcal{T}_{\text {or }} \otimes \mathcal{T}_{\text {or }}$ is defined by the same formula as the skew-symmetrization of (2.1.1), except letting $T_{e}^{2}$ be the subtree that $e$ points to and $T_{e}^{1}$ be the subtree that $e$ points away from (note that there is no pre-Lie comultiplication $\delta_{\mathrm{p}, \mathrm{or}}$ ).

Finally, we will need the generalization given in [Tur05]: Let RTrees, OTrees be the categories whose objects are rooted and oriented trees, respectively, and whose morphisms are embeddings of trees (maps of rooted or oriented trees that preserve incidence and are injective on vertices and edges; the root must get sent to the vertex of the image subtree which is closest to the root of the whole tree).

Definition 2.1.3. For any contravariant functor $\Phi:$ RTrees $\rightarrow$ Sets (called a rooted tree-structure), we let $\mathcal{J}_{\mathrm{rt}}(\Phi)$ be the free $\mathbb{k}$-module spanned by isomorphism classes of pairs $(T, s)$ where $T$ is a rooted tree and $s \in \Phi(T)$. Here an isomorphism of pairs $(T, s) \stackrel{\sim}{\rightarrow}\left(T^{\prime}, s^{\prime}\right)$ is an isomorphism of trees $T \stackrel{\sim}{\rightarrow} T^{\prime}$ whose pullback carries $s^{\prime}$ to $s$. In the oriented case, one similarly defines $\mathcal{T}_{\text {or }}(\Phi)$.

Proposition 2.1.4 ([Tur05]). For any rooted tree-structure $\Phi$, the following formula defines a pre-Lie comultiplication $\delta_{\mathrm{p}, \mathrm{rt}}$ on $\mathcal{T}_{\mathrm{rt}}(\Phi)$ :

$$
\delta_{\mathrm{p}, \mathrm{rt}}(T, s)=\sum_{e \in E(T)}\left(T_{e}^{1},\left.s\right|_{T_{e}^{1}}\right) \otimes\left(T_{e}^{2},\left.s\right|_{T_{e}^{2}}\right),
$$

where $T_{e}^{1} \sqcup T_{e}^{2}=T \backslash\{e\}$ and $T_{e}^{2}$ contains the root. Similarly, the skew-symmetrization of this defines a Lie coalgebra in the case of oriented trees, where now $T_{e}^{2}$ is the tree that e points to.

2.1.3. The Hopf algebra on rooted trees. We briefly recall Connes and Kreimer's Hopf algebra on rooted trees [Kre98], [CK98], as formulated with tree-structures in [Tur05].

Let $\operatorname{Sym}\left(\mathcal{T}_{\mathrm{rt}}\right)$ be the symmetric algebra on $\mathcal{T}_{\mathrm{rt}}$ (polynomials in rooted trees).

Definition 2.1.5 ([CK98]). A cut $H$ of a rooted tree $T$ with root $r t \in V(T)$ is a subset $H \subset E(T)$ of edges. It is a simple cut if each connected component of $T \backslash\{r t\}$ contains at most one edge in $H$. (The empty cut $H=\emptyset$ is included and is simple.)

Definition 2.1.6 ([CK98]). For any simple cut $H$ of $T$, let $\left\{T_{H, e} \mid e \in H\right\} \cup\left\{T_{H, 0}\right\}$ be the set of connected components of $T \backslash H$, where $T_{H, 0}$ is the component containing the root $r t$ and $T_{H, e}$ is the other connected component which was adjacent to $e$ as a subset of $T$. 
Definition 2.1.7 ([CK98], [Tur05]). For any rooted tree-structure $\Phi:$ RTrees $^{\text {op }} \rightarrow$ Sets, define a map $\Delta: \mathcal{T}_{\mathrm{rt}}(\Phi) \rightarrow \operatorname{Sym} \mathcal{T}_{\mathrm{rt}}(\Phi) \otimes \operatorname{Sym} \mathcal{T}_{\mathrm{rt}}(\Phi)$ by the formula

$$
\begin{gathered}
l_{H}(T, s):=\prod_{e \in H}\left(T_{H, e},\left.s\right|_{T_{H, e}}\right) \in \operatorname{Sym} \mathcal{T}_{\mathrm{rt}}(\Phi), \quad r_{H}(T, s)=\left(T_{H, 0},\left.s\right|_{T_{H, 0}}\right), \\
\Delta(T, s)=(T, s) \otimes 1+\sum_{\text {simple cuts } H} l_{H}(T, s) \otimes r_{H}(T, s) .
\end{gathered}
$$

Here, by definition, $l_{\emptyset}(T, s)=1$ and $r_{\emptyset}(T, s)=(T, s)$ for all $T, s$.

Proposition 2.1.8 ([CK98], [Tur05]). This defines a commutative Hopf algebra structure on $\operatorname{Sym} \mathcal{T}_{\mathrm{rt}}(\Phi)$ for any rooted tree-structure $\Phi$, with counit $\varepsilon(X)=0$ for any $X=(T, s)$.

Note that comultiplication has the form (for $X=(T, s)$ )

$$
\Delta(X)=1 \otimes X+X \otimes 1+\Delta^{\prime}(X)
$$

where $\Delta^{\prime}$ is the projection of $\Delta$ away from $\left(1 \otimes \operatorname{Sym} \mathcal{T}_{\mathrm{rt}}(\Phi)\right) \oplus\left(\operatorname{Sym} \mathcal{T}_{\mathrm{rt}}(\Phi) \otimes 1\right)$ (that is, $\Delta^{\prime}=(\mathrm{Id}-\eta \circ \varepsilon)^{\otimes 2} \Delta$ with $\eta$ the unit map).

Furthermore, using the natural grading by total number of edges in the trees, $\Delta^{\prime}(X)$ has strictly lower degree than $X$ if $X$ is a tree. Thus, one may easily verify (cf. [Sch05], §3.9) that the following general formula for the antipode $S$ holds (with $X=(T, s))$ :

$$
S(X)=-X+\sum_{n \geq 1}(-1)^{n+1} \mu^{n} \circ\left(\Delta^{\prime}\right)^{n}(X) .
$$

(Here $\mu^{n}: \operatorname{Sym} \mathcal{T}_{\mathrm{rt}}(\Phi)^{\otimes(n+1)} \rightarrow \operatorname{Sym} \mathcal{T}_{\mathrm{rt}}(\Phi)$ denotes the multiplication and $\left(\Delta^{\prime}\right)^{n}: \operatorname{Sym} \widetilde{\mathcal{T}}_{\mathrm{rt}}(\Phi) \rightarrow \operatorname{Sym} \mathcal{T}_{\mathrm{rt}}(\Phi)^{\otimes(n+1)}$ is the iterated application of the coassociative $\Delta^{\prime}$.) This extends to products of trees anti-multiplicatively. So it is enough to check the bialgebra condition above.

\section{Quiver version and results}

We now proceed to the quiver versions of the preceding and formulate our results.

\subsection{Necklace (pre-)Lie coalgebras}

3.1.1. Original necklace Lie coalgebra ("oriented"). We recall the definition of the necklace Lie coalgebra from [Sch05], which will correspond to the "oriented" case. Let $Q$ be any quiver (with edge set also denoted by $Q$ ) and let $\bar{Q}=Q \sqcup Q^{*}$ be the double quiver, with $Q^{*}:=\left\{e^{*} \mid e \in Q\right\}$, where if $e$ is an arrow from $i$ to $j$ 
(denoted $e: i \rightarrow j$ ), then $e^{*}: j \rightarrow i$ is the reverse. The double quiver has the same set $I$ of edges as $Q$. Let $P$ be the path algebra on the double quiver. Precisely, one has $P=T_{\mathbb{k}} I\langle\bar{Q}\rangle$, where $\langle\bar{Q}\rangle$ is the $\mathbb{k}^{I}$-bimodule with basis $\bar{Q}$, so that $i\langle\bar{Q}\rangle j$ is the space with basis those edges $e: i \rightarrow j$. For each edge $e: i \rightarrow j$ let $e_{s}:=i, e_{t}:=j$ (the "source" and "target", respectively).

Let $L:=P /[P, P]$ be the $\mathbb{k}$-module with basis the cyclic paths in the quiver $\bar{Q}$ (forgetting the initial edge). We call such cyclic paths "necklaces," and the cobracket operation will involve splitting necklaces into two necklaces (by making two cuts and gluing the endpoints of the two resulting strands $=$ paths in the quiver). Then one defines the cobracket $\delta=\delta_{\text {or }}: L \rightarrow L \wedge L$ (or $=$ oriented) as follows:

$$
\delta_{\mathrm{or}}\left(\left[a_{1} \ldots a_{n}\right]\right)=\sum_{i<j} \omega\left(a_{i}, a_{j}\right)\left[\left(a_{j}\right)_{t} a_{j+1} \ldots a_{i-1}\right] \wedge\left[\left(a_{i}\right)_{t} a_{i+1} \ldots a_{j-1}\right],
$$

where $\omega\left(e, e^{*}\right)=-\omega\left(e^{*}, e\right)=1$ for $e \in Q$, and $\omega(e, f)=0$ if $e \neq f^{*}$ (we use the notation $\left(e^{*}\right)^{*}:=e$ ). A typical summand is depicted in Figure 1.

Before:
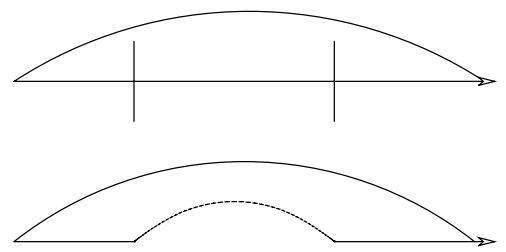

After:

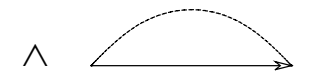

Figure 1. The original Lie cobracket on $P /[P, P]$ from [Sch05].

3.1.2. Necklace (pre-)Lie coalgebra of paths, "rooted". We define "rooted" versions of the necklace Lie coalgebra. By being rooted, we will actually obtain a pre-Lie structure, as in the rooted tree case.

To add a basepoint to a necklace, one should pick an initial edge. Equivalently, one can replace the necklace with a closed path. In this generality, one may actually speak of non-closed paths as well, which we do.

In [Sch07], a (Loday or Lie) cobracket is defined in this way by the following idea: When one makes two cuts in a path and joins the cut ends the same way we did before for necklaces, one obtains one path and one necklace. This is depicted in Figure 2. This will not give a pre-Lie coalgebra, however: to get one, one needs (as in [Tur05]) to split a path into two paths (resp. a rooted tree into two rooted trees by cutting). To do this, we make two cuts in the path, but only glue once: the left strand to the right, obtaining two strands (Figure 3). Precisely, we define the rooted pre-Lie 
Before:
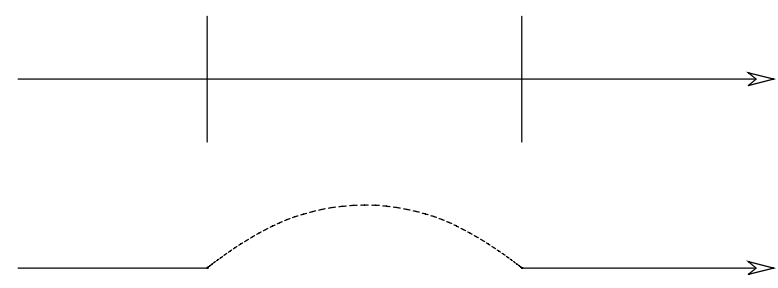

After:

$\otimes$

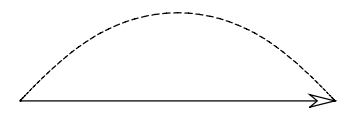

Figure 2. The Loday cobracket $P \rightarrow P /[P, P] \otimes P$ from [Sch07].

Before:
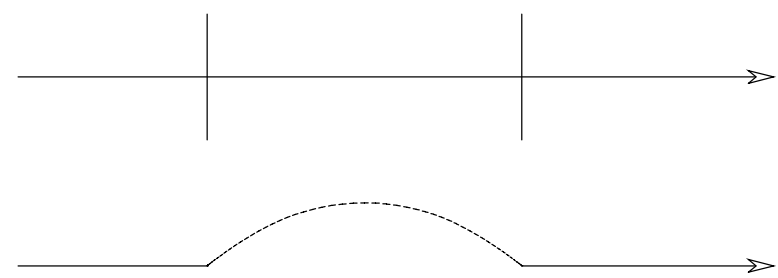

After:

$\otimes$

Figure 3. A summand in the pre-Lie multiplication of "Before".

comultiplication $\delta_{\mathrm{p}, \mathrm{rt}}$ and Lie cobracket $\delta_{\mathrm{rt}}$ by the formulas

$$
\begin{gathered}
\delta_{\mathrm{p}, \mathrm{rt}}\left(a_{1} \ldots a_{n}\right)=\sum_{i<j}-\omega\left(a_{i}, a_{j}\right)\left(a_{i}\right)_{t} a_{i+1} \ldots a_{j-1} \otimes\left(a_{1}\right)_{s} a_{1} \ldots a_{i-1} a_{j+1} \ldots a_{n}, \\
\delta_{\mathrm{rt}}=\delta_{\mathrm{p}, \mathrm{rt}}-\tau_{(12)} \delta_{\mathrm{p}, \mathrm{rt}} .
\end{gathered}
$$

Proposition 3.1.2. The maps $\delta_{\mathrm{or}}$, $\delta_{\mathrm{rt}}$ are Lie cobrackets, and $\delta_{\mathrm{p}, \mathrm{rt}}$ is a pre-Lie comultiplication.

Proof. It suffices to check that $\delta_{\mathrm{p}, \mathrm{rt}}$ is a pre-Lie comultiplication $\left(\delta_{\mathrm{or}}\right.$ is a Lie cobracket by [Sch05]). This follows along similar lines to the proof that $\delta_{\text {or }}$ is a Lie cobracket in [Sch05], §2.2.

Note that, while in the rooted case, one may consider paths that are not closed, the first component of the image of $\delta_{\mathrm{p}, \mathrm{rt}}$ lies in the span of closed paths. 
3.2. Hopf algebra of paths. We now define a Hopf algebra which completes the analogy "pre-Lie coalgebra of rooted trees :: Renormalization Hopf algebra $==$ Necklace pre-Lie coalgebra of paths (rooted) :: ??" Note that this does not have an oriented version (since there is no pre-Lie coalgebra in the oriented case, cf. Proposition 3.5.2).

Definition 3.2.1. Given a path $a_{1} \ldots a_{n} \in P$, a cut $H$ is a choice of pairs

$$
H=\left\{\left(i_{1}, j_{1}\right), \ldots,\left(i_{m}, j_{m}\right)\right\} \subset\{1, \ldots, n\}^{2}
$$

such that

(1) $i_{1}, j_{1}, \ldots, i_{m}, j_{m}$ are distinct,

(2) for all $\ell, i_{\ell}<j_{\ell}$,

(3) the pairs do not cross: that is, there do not exist $\ell, \ell^{\prime}$ such that $i_{\ell}<i_{\ell^{\prime}}<j_{\ell}<j_{\ell^{\prime}}$, and

(4) for all $\ell$, there exists $e_{\ell} \in Q$ such that $\left\{a_{i_{\ell}}, a_{j_{\ell}}\right\}=\left\{e_{\ell}, e_{\ell}^{*}\right\}$.

Definition 3.2.2. A cut is called simple if there do not exist $\ell, \ell^{\prime}$ with $i_{\ell}<i_{\ell^{\prime}}<j_{\ell^{\prime}}<$ $j_{\ell}$ : that is, as in Figure 4, no added (semicircular) edge contains another such.

Definition 3.2.3. For any cut $H$ of a path $X:=a_{1} \ldots a_{n}$, let $\left\{X_{H, c}\right\}_{c \in H} \sqcup\left\{X_{H, 0}\right\}$ be the collection of paths obtained by applying Figure 3 repeatedly (cut each pair of edges $a_{i_{\ell}}, a_{j_{\ell}}$ and glue one pair of endpoints each, as in (3.1.1)). By definition, $X_{H, 0}$ is the unique path which shares the endpoints of the original path (beginning at $\left(a_{1}\right)_{s}$ and ending at $\left.\left(a_{n}\right)_{t}\right)$, and $X_{H, c}$ for $c=\left(i_{\ell}, j_{\ell}\right)$ is the unique path which begins at the target $\left(a_{i_{\ell}}\right)_{t}$ of $a_{i_{\ell}}$ and ends at the source $\left(a_{j_{\ell}}\right)_{s}$ of $a_{j_{\ell}}$.

Definition 3.2.4. For any cut $H=\left\{\left(i_{1}, j_{1}\right), \ldots,\left(i_{m}, j_{m}\right)\right\}$ of a path $a_{1} \ldots a_{n}$, let $\varepsilon_{H}= \pm 1$ be defined by

$$
\varepsilon_{H}:=\prod_{\ell=1}^{m}-\omega\left(a_{i_{\ell}}, a_{j_{\ell}}\right) .
$$

Definition 3.2.5. Define the coproduct $\Delta: \operatorname{Sym} P \rightarrow(\operatorname{Sym} P)^{\otimes 2}$ on a path $X=$ $a_{1} \ldots a_{n}$ by

$$
\begin{aligned}
l_{H}(X) & :=X_{H, c_{1}} \& \ldots \& X_{H, c_{|H|}}, \quad r_{H}(X):=X_{H, 0}, \\
\Delta(X) & :=X \otimes 1+\sum_{\text {simple cuts } H} \varepsilon_{H} l_{H}(X) \otimes r_{H}(X),
\end{aligned}
$$

where $H=\left\{c_{1}, \ldots, c_{|H|}\right\}$. 
Proposition 3.2.8. The map $\Delta$ endows $\operatorname{Sym} P$ with the structure of a commutative Hopf algebra with antipode given by (2.1.9) (where $X$ is a path).

This proposition will be proved in Section 4.1. Note that the result also follows from Theorem 3.3.16, since the map $\eta$ gives a monomorphism of Hopf algebras (i.e., without knowing Sym $P$ is Hopf, the theorem shows that $\eta$ is injective and carries the proposed multiplication, comultiplication, unit, and counit to those for the ConnesKreimer Hopf algebra).

\subsection{The monomorphisms to renormalization algebras.}

3.3.1. The tree-structures. Following in analogy with [Tur05], we define a preLie coalgebra map $\left(P, \delta_{\mathrm{p}, \mathrm{rt}}\right) \rightarrow\left(\mathcal{T}_{\mathrm{rt}}\left(\Phi_{\mathrm{rt}}\right), \delta_{\mathrm{p}, \mathrm{rt}}\right)$ and a Lie coalgebra map $\left(L, \delta_{\mathrm{or}}\right) \rightarrow$ $\left(\mathcal{T}_{\text {or }}\left(\Phi_{\text {or }}\right), \delta_{\text {or }}\right)$ for certain rooted (resp. oriented) tree-structures $\Phi_{\mathrm{rt}}, \Phi_{\mathrm{or}}$. The former induces a Hopf algebra map $(\operatorname{Sym}(P), \Delta) \rightarrow\left(\operatorname{Sym}\left(\mathcal{T}_{\mathrm{rt}}\right), \Delta\right)$, as we explain. As a result of the pre-Lie coalgebra map, one also obtains a Lie coalgebra map on the associated Lie coalgebra $\left(P, \delta_{\mathrm{rt}}\right)$ (by skew-symmetrizing the pre-Lie comultiplication).

Definition 3.3.1. A ribbon graph structure on a tree is a choice, for each vertex of the tree, of a fixed cyclic ordering of the edges incident with that vertex.

Definition 3.3.2. Let $\Phi_{\text {or }}$ be the oriented tree-structure which assigns to each oriented tree a choice of ribbon graph structure on the tree, and to each vertex of the tree a cyclic path in $\bar{Q}$ (which is a basis element of $L$ ).

Definition 3.3.3 ([Tur05]). A corner $^{3}$ of a vertex of a ribbon graph is a choice of initial edge at the vertex (giving a linear ordering of the incident edges).

Definition 3.3.4. Let $\Phi_{\mathrm{rt}}$ be the rooted tree-structure which assigns to each rooted tree a choice of ribbon graph structure with a corner at the root, together with an orientation of all edges and a labeling of vertices by paths in $\bar{Q}$ (basis elements of $P$ ).

Remark 3.3.5. Instead of assigning (cyclic) paths to each vertex, an alternative would be to assign a single element of $P^{\otimes V}$ or $L^{\otimes V}$ to the tree, where $\otimes V$ means taking $\otimes|V|$ with components labeled by $V$. Then $\Phi_{*}$ would obtain a $\mathbb{k}$-module structure, and we could work with the quotient $\overline{\mathcal{T}\left(\Phi_{*}\right)}$ of $\mathcal{T}\left(\Phi_{*}\right)$ by the relation $\left(T, s+s^{\prime}\right)=$ $(T, s)+\left(T, s^{\prime}\right)$.

Remark 3.3.6. Note that at all vertices other than the root of a rooted tree with a ribbon-graph structure, the cyclic ordering actually has a canonical lifting to a linear

\footnotetext{
${ }^{3}$ This is called a corner to agree with [Tur05], Remark 2 of $\$ 5.2$, where it is defined as a choice of two consecutive edges in the cyclic ordering (these are the last and first edges in our linear ordering).
} 
ordering (i.e., a corner) by choosing as initial the edge that lies between the given vertex and the root. So with the corner at the root, one obtains rooted trees with linear orderings at all vertices (rather than merely cyclic orderings).

3.3.2. Chord diagrams and dual trees. In order to define the homomorphisms of (pre-)Lie coalgebras and Hopf algebras, we first need to construct from a simple cut of a (cyclic) path the dual tree to the chord diagram associated to this cut:

Definition 3.3.7. For any path $X=a_{1} \ldots a_{n}$ of length $n$, associate to this a line segment $L_{X} \subset \mathbb{R}$ with edges $e_{i}=\left[i-\frac{1}{2}, i+\frac{1}{2}\right]$ for all $1 \leq i \leq n$, and vertices $\left\{\frac{1}{2}, \frac{3}{2}, \ldots, n+\frac{1}{2}\right\}$. The root is defined to be $r t:=\frac{1}{2}$. (See Figure 4).

We also think of the vertex $n+\frac{1}{2}$ as the root, essentially considering it to be the same vertex as $\frac{1}{2}$. That is, a circle with basepoint is essentially equivalent to a line by cutting at the basepoint: then the endpoints of the line are both the basepoint. We chose $\frac{1}{2}$ rather than $n+\frac{1}{2}$ for the root only for definiteness: the choice makes no difference.

Definition 3.3.8. For any cut $H=\left\{\left(i_{1}, j_{1}\right), \ldots,\left(i_{m}, j_{m}\right)\right\}$ of a path $X=a_{1} \ldots a_{n} \in$ $P$, consider the associated chord diagram $C_{X, H}$, obtained from $L_{X}$ by adding interior vertices $i_{\ell}, j_{\ell}$ to the edges $e_{i_{\ell}}, e_{j_{\ell}}$, and a new edge with endpoints $i_{\ell}, j_{\ell}$ for each $\ell$, as in Figure 4. The edges are chosen so as to not intersect (giving a planar graph).

Definition 3.3.9. For any cut $H$ as above, let the dual rooted tree $T_{H}$ be obtained by dualizing the chord diagram: place one vertex inside each face of the chord diagram, and one edge crossing each edge of the chord diagram, connecting the vertices associated to the two faces. The root corresponds to the unbounded face (which is included as a face).

Definition 3.3.10. For any cut $H$ of a path as above with dual rooted tree $T_{H}$, we define an element $s_{H}$ of $\Phi_{\mathrm{rt}}\left(T_{H}\right)$ as follows: first, orient the edges of the chord diagram, by assigning the edge with endpoints $i_{\ell}, j_{\ell}$ the orientation $i_{\ell} \rightarrow j_{\ell}$ if $a_{i_{\ell}} \in Q$ and $j_{\ell} \rightarrow i_{\ell}$ otherwise. Then the orientation of the edge $e$ of $T_{H}$ which crosses an edge $f$ of the chord diagram is such that $e \wedge f$ is the positive orientation on $\mathbb{R}^{2}$. Next, the unbounded face is still considered a face and its vertex is declared the root. This is naturally a ribbon graph. The linear ordering of the edges at the root (choice of corner) is given by the usual linear ordering of the endpoints of the edges of the unbounded face of the chord diagram in the interval $\left[\frac{1}{2}, n+\frac{1}{2}\right]$. Finally, the labeling of the vertices is given as follows: to each face $f$ of the chord diagram, $J=\partial f \cap\left[\frac{1}{2}, n+\frac{1}{2}\right]$ is a union of closed intervals; let $J^{\circ}$ be the interior. Let $i_{J}$ be the vertex of the quiver which is the left endpoint of $J$ : that is, $i_{J}=\left(a_{\min \left(J^{\circ} \cap\{1, \ldots, n\}\right)}\right) t$. Then $i_{J} \prod_{j \in J^{\circ} \cap\{1,2, \ldots, n\}} a_{j}$ is the path associated to the vertex attached to $f$ (it is the path which remains in that face after performing cuts as in Figure 4). 
The dual tree is depicted (without the tree-structure) in Figure 4.

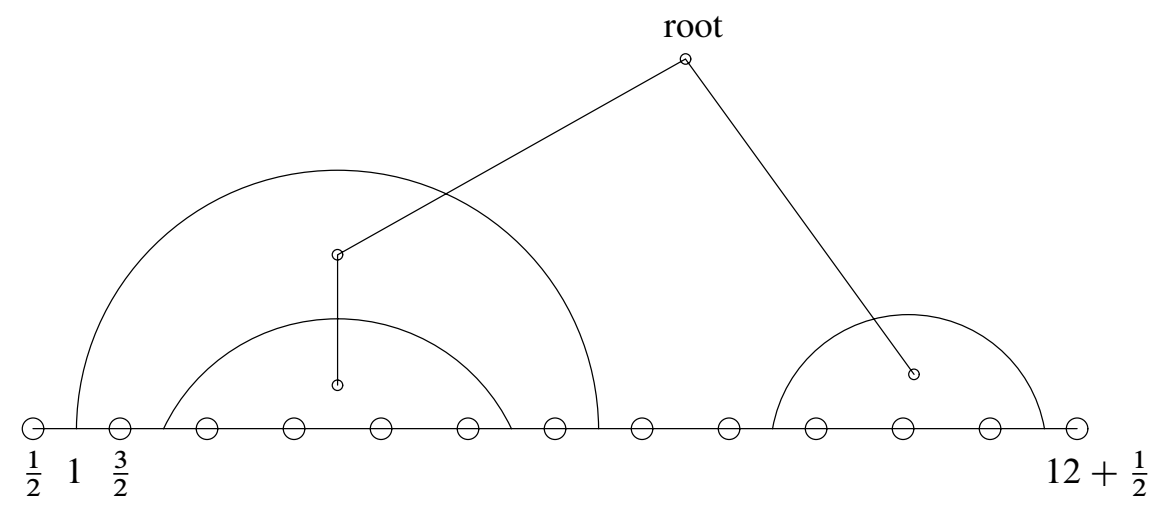

Figure 4. A typical chord diagram (for a cut) and its dual tree.

We proceed to define the dual oriented tree.

Definition 3.3.11. For any cut $H$ as above, the dual oriented tree is the dual tree $T_{H}$, forgetting the root, together with the orientation of edges given by the element of $\Phi_{\mathrm{rt}}\left(T_{H}\right)$ above. Call it $T_{H \text {,or }}$.

Definition 3.3.12. For any closed path $a_{1} \ldots a_{m}$ with dual oriented tree $T_{H \text {,or }}$, define an element $s_{H \text {,or }}$ of $\Phi_{\text {or }}\left(T_{H, \text { or }}\right)$ from $s_{H}$ by taking the image of the labels of vertices in cyclic paths $(L=P /[P, P])$ and forgetting the corner structure.

The following is easily verified.

Lemma 3.3.13. For any closed path $a_{1} \ldots a_{m}$, the space of cuts of $a_{1} \ldots a_{m}$ is naturally isomorphic to that of $a_{i} a_{i+1} \ldots a_{m} a_{1} \ldots a_{i-1}$, for all $i$, in a way that yields a natural isomorphism of dual oriented trees which carries the elements $\Phi_{\mathrm{or}}\left(T_{H, \mathrm{or}}\right)$ to each other. One may therefore define a cut of the cyclic path $\left[a_{1} \ldots a_{m}\right]$ and its associated dual oriented tree $T_{H, \mathrm{or}}$, with associated element $s_{H, \mathrm{or}} \in \Phi_{\mathrm{or}}\left(T_{H, \mathrm{or}}\right)$.

3.3.3. The homomorphisms. Finally, we may define the homomorphisms and state the first theorem.

Definition 3.3.14. For any path $a_{1} \ldots a_{n}$, define the element $\eta_{\mathrm{rt}}\left(a_{1} \ldots a_{n}\right) \in \mathcal{T}_{\mathrm{rt}}\left(\Phi_{\mathrm{rt}}\right)$ by

$$
\eta_{\mathrm{rt}}\left(a_{1} \ldots a_{n}\right)=\sum_{\text {cuts } H} \varepsilon_{H}\left(T_{H}, s_{H}\right) .
$$


Definition 3.3.15. For any cyclic path $\left[a_{1} \ldots a_{n}\right]$, define the element $\eta_{\mathrm{or}}\left(\left[a_{1} \ldots a_{n}\right]\right) \in$ $\mathcal{T}_{\text {or }}\left(\Phi_{\text {or }}\right)$ by

$$
\eta_{\text {or }}\left(\left[a_{1} \ldots a_{n}\right]\right)=\sum_{\text {cuts } H}\left(T_{H, \text { or }}, s_{H, \text { or }}\right) .
$$

Theorem 3.3.16. The maps $\eta_{\mathrm{or}}$ and $\eta_{\mathrm{rt}}$ extend linearly to an injective pre-Lie coalgebra homomorphism $\left(P, \delta_{\mathrm{p}, \mathrm{rt}}\right) \rightarrow \mathcal{T}_{\mathrm{rt}}\left(\Phi_{\mathrm{rt}}\right)$ and an injective Lie coalgebra homomorphism $\left(L, \delta_{\mathrm{or}}\right) \rightarrow \mathcal{T}_{\mathrm{or}}\left(\Phi_{\mathrm{or}}\right)$, respectively. Furthermore, $\eta_{\mathrm{rt}}$ extends multiplicatively (and linearly) to a Hopf algebra monomorphism $\operatorname{Sym} P \rightarrow \operatorname{Sym} \mathcal{T}_{\mathrm{rt}}\left(\Phi_{\mathrm{rt}}\right)$.

This theorem will be proved in Section 4.2.

M. Livernet pointed out to us that, by [CL01], the pre-Lie coalgebra of decorated trees $\mathcal{T}_{\mathrm{rt}}(\Phi)$ is a cofree pre-Lie coalgebra for any $\Phi$. This explains why morphisms such as the above must always exist (although the one we construct is particularly natural).

3.4. Factorization of $\eta$ through chord algebras. It turns out that one can understand the $\eta$ homomorphisms (and their injectivity) through a factorization as follows:

$$
\begin{gathered}
\left(L, \delta_{\mathrm{or}}\right) \stackrel{S_{\mathrm{or}}}{\longrightarrow} C h_{\mathrm{or}} \stackrel{D_{\mathrm{or}}}{\longrightarrow} \mathcal{T}_{\mathrm{or}}\left(\Phi_{\mathrm{or}}\right), \\
\left(P, \delta_{\mathrm{p}, \mathrm{rt}}\right) \stackrel{S_{\mathrm{rt}}}{\longrightarrow} C h_{\mathrm{rt}} \stackrel{D_{\mathrm{rt}}}{\longrightarrow} \mathcal{T}_{\mathrm{rt}}\left(\Phi_{\mathrm{rt}}\right),
\end{gathered}
$$

where $C h_{\mathrm{or}}$ and $C h_{\mathrm{rt}}$, called chord algebras, are spanned by chord diagrams on necklaces or paths, and the first maps in (3.4.1), (3.4.2) take a necklace (resp., path) to the sum of all possible chord diagrams on that necklace or path. We will equip $C h_{\mathrm{rt}}$ and $C h_{\text {or }}$ with the appropriate pre-Lie and Lie coalgebra structures, and Sym $C h_{\mathrm{rt}}$ with the appropriate Hopf algebra structure, so that one obtains the following sequence of Hopf algebra homomorphisms:

$$
\operatorname{Sym} P \stackrel{S_{\mathrm{rt}}}{\longrightarrow} \operatorname{Sym} C h_{\mathrm{rt}} \stackrel{D_{\mathrm{rt}}}{\longrightarrow} \operatorname{Sym} \mathcal{T}_{\mathrm{rt}}\left(\Phi_{\mathrm{rt}}\right) .
$$

We also briefly explain how this construction can also be done in the context of [Tur05], yielding the space of geometric chord diagrams.

Definition 3.4.4. A necklace chord diagram is a necklace (= cyclic monomial) $\left[a_{1} \ldots a_{n}\right] \in L$, for $a_{i} \in \bar{Q}$, together with a cut $H$ of $\left[a_{1} \ldots a_{n}\right]$. Denote the necklace chord diagram by $\left(\left[a_{1} \ldots a_{n}\right], H\right)$.

Definition 3.4.5. A path chord diagram is a path $a_{1} \ldots a_{n} \in P$ for $a_{i} \in \bar{Q}$ together with a cut $H$ of $a_{1} \ldots a_{n}$. The path chord diagram is denoted by $\left(a_{1} \ldots a_{n}, H\right)$. 
Definition 3.4.6. The chord algebra $C h_{\mathrm{rt}}$ is defined(as a linear space) to be the free $\mathbb{k}$-module with basis the path chord diagrams. Similarly, $C h_{\text {or }}$ is defined to have basis the necklace chord diagrams.

Definition 3.4.7. Define $\delta_{\mathrm{p}, \mathrm{rt}}: C h_{\mathrm{rt}} \rightarrow C h_{\mathrm{rt}}^{\otimes 2}$ as follows. Let $X=a_{1} \ldots a_{n}$ be a path for $a_{\ell} \in \bar{Q}$, and let $H=\left\{\left(i_{1}, j_{1}\right), \ldots,\left(i_{m}, j_{m}\right)\right\}$ be a cut. For any $1 \leq$ $\ell \leq m$, let $c_{\ell}:=\left(i_{\ell}, j_{\ell}\right)$ and let $H_{c_{\ell}}^{1}, H_{c_{\ell}}^{2} \subset H$ be the two subcuts obtained by removing $c_{\ell}=\left(i_{\ell}, j_{\ell}\right)$ from $H: H_{c_{\ell}}^{1}$ is the collection of $\left(i_{\ell^{\prime}}, j_{\ell^{\prime}}\right)$ on the inside of $c_{\ell}$ (in particular, $\ell^{\prime} \neq \ell$ ), and $H_{c_{\ell}}^{2}$ is the collection on the outside of $c_{\ell}$. Explicitly, $\left(i_{\ell^{\prime}}, j_{\ell^{\prime}}\right) \in H_{c \ell}^{1}$ iff $i_{\ell^{\prime}}>i_{\ell}$ (equivalently, $j_{\ell^{\prime}}<j_{\ell}$ ). Finally, we then let $X_{c_{\ell}, 1}, X_{c_{\ell}, 2}$ be two chord diagrams thus obtained: $X_{c_{\ell}, 1}:=\left(\left(a_{i_{\ell}}\right)_{t} a_{i_{\ell}+1} \ldots a_{j_{\ell}-1}, H_{c_{\ell}}^{1}\right)$ and $X_{c_{\ell}, 2}:=\left(a_{1} \ldots a_{i_{\ell}-1}\left(a_{j_{\ell}}\right)_{t} a_{j_{\ell}+1} \ldots a_{n}, H_{c_{\ell}}^{2}\right)$. Then we define

$$
\delta_{\mathrm{p}, \mathrm{rt}}(X, H)=\sum_{\ell=1}^{m}-\omega\left(a_{i_{\ell}}, a_{j_{\ell}}\right) X_{c_{\ell}, 1} \otimes X_{c_{\ell}, 2} .
$$

Definition 3.4.8. Define $\delta_{\mathrm{or}}: C h_{\mathrm{or}} \rightarrow C h_{\mathrm{or}}^{\otimes 2}$ as follows. Let $X=\left[a_{1} \ldots a_{n}\right]$ be a necklace for $a_{\ell} \in \bar{Q}$, and let $H$ be a cut of $X$, which corresponds to the cut $\left\{\left(i_{1}, j_{1}\right), \ldots,\left(i_{m}, j_{m}\right)\right\}$ of $a_{1} \ldots a_{n}$. For any $1 \leq \ell \leq m$, let $X_{c_{\ell}, 1}, X_{c_{\ell}, 2}$ be the two chord diagrams obtained by removing $\left(i_{\ell}, j_{\ell}\right)$ from $H$, defined as in Definition 3.4.7, except adding braces $[\cdot]$ around the obtained paths. Then define

$$
\delta_{\text {or }}(X, H)=\sum_{\ell=1}^{m}-\omega\left(a_{i_{\ell}}, a_{j_{\ell}}\right)\left(X_{c_{\ell}, 1} \otimes X_{c_{\ell}, 2}-X_{c_{\ell}, 2} \otimes X_{c_{\ell}, 1}\right) .
$$

Definition 3.4.9. Define the coproduct $\Delta$ on Sym $C h_{\mathrm{rt}}$ as follows: For any chord diagram $X:=\left(a_{1} \ldots a_{n}, H\right)$ with $H=\left\{\left(i_{1}, j_{1}\right), \ldots,\left(i_{m}, j_{m}\right)\right\}$, and any simple cut $H^{\prime}$ with $H^{\prime} \subset H$, let $\left\{X_{H^{\prime}, c}\right\}_{c \in H^{\prime}} \cup\left\{X_{H^{\prime}, 0}\right\}$ be the collection of chord diagrams obtained by cutting out the chords in $H^{\prime}$ : each time we cut out a chord from $H^{\prime}$, we divide a cut into two separate cuts, as in Definition 3.4.7, and divide the corresponding path into two paths. Then $X_{H^{\prime}, 0}$ is the obtained chord diagram which contains the basepoint $\left(\left(a_{1}\right)_{s}\right.$ and $\left.\left(a_{n}\right)_{t}\right)$, and $\left\{X_{H^{\prime}, c}\right\}$ is the other chord diagram which is cut from the chord $c \in H^{\prime}$. Then we define

$$
\begin{gathered}
l_{H^{\prime}}(X):=\prod_{c \in H^{\prime}} X_{H^{\prime}, c}, \quad r_{H^{\prime}}(X)=X_{H^{\prime}, 0}, \quad \varepsilon_{H^{\prime}}=\prod_{c=\left(i_{\ell}, j_{\ell}\right) \in H}-\omega\left(a_{i_{\ell}}, a_{j_{\ell}}\right), \\
\Delta\left(\left(a_{1} \ldots a_{n}, H\right)\right)=X \otimes 1+\sum_{\text {simple cuts } H^{\prime} \subset H} \varepsilon_{H^{\prime}} l_{H^{\prime}}(X) \otimes r_{H^{\prime}}(X) .
\end{gathered}
$$

Finally we have the following theorem, which is a strengthening of Theorem 3.3.16. 
Theorem 3.4.10. The algebra $\left(C h_{\mathrm{rt}}, \delta_{\mathrm{p}, \mathrm{rt}}\right)$ is a pre-Lie coalgebra and $\left(C h_{\mathrm{or}}, \delta_{\mathrm{or}}\right)$ is a Lie coalgebra, and Sym $C h_{\mathrm{rt}}$ is a Hopf algebra given by (3.4.9), (2.1.9) (with counit given by $\varepsilon(X)=0$ for any chord diagram $X$ ). Then the diagrams (3.4.1),(3.4.2) and (3.4.3) are homomorphisms, where $S_{*}$ takes a path or necklace to the sum over all chord diagrams over that path or necklace, and $D_{*}$ takes a chord diagram with cut $H$ to its dual tree in the oriented case, and to $\varepsilon_{H}$ times its dual tree in the rooted case, assigning data as in Section 3.3.3.

This theorem will be proved in Section 4.2.

3.4.1. Geometric chord diagrams. We briefly indicate the geometric counterpart of Theorem 3.4.10 (since algebras of chord diagrams were not discussed in [Tur05]). We consider geometric chord diagrams with a single loop: this means ([AMR96]) a smooth map of a loop with chords into a surface such that the map is constant on the chords. Let us also assume that the chords are such that, if the loop is drawn in the plane and all the chords are in the interior of the loop, then the chords do not intersect. Then we let $C h_{\mathrm{rt}}$ be the algebra which, as a free $\mathbb{k}$-module, has basis the isotopy classes of geometric chord diagrams with a single loop with basepoint (the isotopies must be through such geometric chord diagrams), and $C h_{\text {or }}$ is, as a $\mathbb{k}$-module, the space of isotopy classes of geometric chord diagrams with a single loop without basepoint. One may then form the sequences of homomorphisms (3.4.1), (3.4.2) and (3.4.3): the map $S_{*}$ takes an isotopy class of loops to the sum of all isotopy classes of chord diagrams whose underlying isotopy class of loops is the original class, and the map $D_{*}$ takes a geometric chord diagram to the dual tree, which then has all the structure required of $\Phi_{\mathrm{or}}, \Phi_{\mathrm{rt}}$.

3.5. Relationship between the Hopf algebras and pre-Lie algebras. We note that the Hopf algebras considered here all have the following special form: as an algebra they are Sym $V$ for some vector space $V$, and the comultiplication $\Delta$ and counit $\varepsilon$ have the form

$$
\Delta(v)=v \otimes 1+1 \otimes v+\Delta^{\prime}(v), \quad \Delta^{\prime}(v) \in\left(\operatorname{Sym}^{\geq 1} V\right) \otimes V, \quad \varepsilon(v)=0
$$

for all $v \in V$. We claim that such Hopf algebras are in one-to-one correspondence with pre-Lie comultiplications on the vector space $V$ as follows (this was essentially observed in [OG05] in the dual setting, but not quite formulated in the same way):

Proposition 3.5.2. Let $V$ be any $\mathbb{Z}_{+}$-graded vector space.

(i) For any Hopf algebra structure on $\operatorname{Sym} V$ satisfying (3.5.1), the map $\rho: V \rightarrow$ $V \otimes V$ given by the composition of $\Delta^{\prime}$ with the projection to $V \otimes V$, is a pre-Lie comultiplication.

(ii) Conversely, given any pre-Lie comultiplication $\rho: V \rightarrow V \otimes V$ which preserves the total grading (induced by the grading on $V$ ), there exists a unique graded comultiplication on Sym $V$ of the form (3.5.1), which yields $\rho$ as in part (i). 
(iii) Moreover, we describe an inductive procedure for computing the $\Delta$ guaranteed in part (ii).

Proof. In all parts, let subscripts ${ }_{n}$ denote the standard (not total) grading on Sym $V$ so that $(\operatorname{Sym} V)_{n}=\operatorname{Sym}^{n} V$, extended to $(\operatorname{Sym} V)^{\otimes 2}$ and $(\operatorname{Sym} V)^{\otimes 3}$ by

$$
\begin{aligned}
& (\operatorname{Sym} V)_{n}^{\otimes 2}=\bigoplus_{i+j=n} \operatorname{Sym}^{i} V \otimes \operatorname{Sym}^{j} V \\
& (\operatorname{Sym} V)_{n}^{\otimes 3}=\bigoplus_{i+j+k=n} \operatorname{Sym}^{i} V \otimes \operatorname{Sym}^{j} V \otimes \operatorname{Sym}^{k} V .
\end{aligned}
$$

(i) Let us formally write

$$
\Delta=\Delta_{0}+\Delta_{1}+\Delta_{2}+\cdots,
$$

where $\Delta_{n}: V \rightarrow \operatorname{Sym}^{n} V \otimes V$ for $n \geq 1$ and $\Delta_{0}(v)=1 \otimes v+v \otimes 1$ for any $v \in V$ (and $\Delta_{n}(1)=0$ if $n>0$ and $\Delta_{0}(1)=1 \otimes 1$ ); for any $v \in V$, only finitely many of the $\Delta_{n}(v)$ can be nonzero. Also, let $\Delta_{0}^{\prime}(X):=\Delta_{0}(X)-(1 \otimes X+X \otimes 1)$ (thus $\Delta_{0}^{\prime}\left(v_{1} v_{2}\right)=v_{1} \otimes v_{2}+v_{2} \otimes v_{1}$ for any $\left.v_{1}, v_{2} \in V\right)$. Then, modulo $\bigoplus_{m \geq 4}(\operatorname{Sym} V)_{m}^{\otimes 3}$, one has

$$
\begin{aligned}
0 & =(\Delta \otimes 1) \Delta(v)-(1 \otimes \Delta) \Delta(v) \\
& =\left(\left(\Delta_{1} \otimes 1\right) \Delta_{1}(v)-\left(1 \otimes \Delta_{1}\right) \Delta_{1}(v)\right)+\left(\Delta_{0}^{\prime} \otimes 1\right) \Delta_{2}(v) .
\end{aligned}
$$

This equation says that, setting $\rho:=\Delta_{1}, \rho$ is pre-Lie (since $\left(\Delta_{0}^{\prime} \otimes 1\right) \Delta_{2}(v)$ is symmetric in the first and second components).

(ii), (iii) Suppose that we are given $\Delta_{1}, \ldots, \Delta_{n}$ for $n \geq 1$ such that $\Delta_{\leq n}:=\Delta_{0}+\Delta_{1}+\cdots+\Delta_{n}$, extended multiplicatively to Sym $V$, is coassociative modulo $\bigoplus_{m \geq n+2}(\operatorname{Sym} V)_{m}^{\otimes 3}$. We would like to find $\Delta_{n+1}$ such that $\Delta_{\leq n}+\Delta_{n+1}$ is coassociative modulo $\bigoplus_{m \geq n+3}(\operatorname{Sym} V)_{m}^{\otimes 3}$. This is equivalent to

$$
\left(\Delta_{0}^{\prime} \otimes 1\right) \Delta_{n+1}(v)=\sum_{i+j=n+1, i, j>0}\left(1 \otimes \Delta_{i}-\Delta_{i} \otimes 1\right) \Delta_{j}(v) .
$$

In particular, $\Delta_{n+1}$ exists iff the first two components live in $\Delta_{0}^{\prime}(\operatorname{Sym} V)$, and in this case $\Delta_{n+1}$ is unique and may be computed algorithmically as indicated. Existence follows from the dual version of the construction of [OG05].

We note that we used the grading above only to guarantee finiteness of the sum $\Delta_{0}+\Delta_{1}+\cdots$ on any vector $v \in V$ : without assuming gradedness, the above proposition still holds if we work in the completed tensor product $\operatorname{Sym} V \otimes \operatorname{Sym} V$ with respect to the grading $(\operatorname{Sym} V)_{\bullet}^{\otimes 2}$. Alternatively, one could assume that iterated applications of $\rho$ on any $v \in V$ eventually yield zero.

As a result of the proposition, proving the main results of this paper (or [Tur05]) on the pre-Lie level is in fact equivalent to proving them on the Hopf algebra level, 
as one can translate between the two using the above proposition. This explains why one must arrive at (3.2.7) (resp. the formulas from [Tur05], §8.3) for coproduct given the choice of pre-Lie comultiplication.

We see that the fact that the pre-Lie structure fails to exist in the oriented case is the same as the fact that the renormalization Hopf algebra does not exist without using rooted trees.

3.5.1. Noncommutative version. In [Tur05], §8.5, a "noncommutative" version of the Hopf algebras was defined, using the tensor algebras over $V$ instead of the symmetric algebras. In this version, $\Delta_{0}$ is the usual "shuffle" coproduct on $T(V)$ generated multiplicatively by $\Delta_{0}(v)=1 \otimes v+v \otimes 1$ for $v \in V$. In this case, Proposition 3.5.2 is no longer true (for instance, $\Delta_{0}^{\prime}(v w-w v)=0$ for any $v, w \in V$, so that one would have a choice of $\Delta_{2}$ ).

However, in the case of paths, as in Turaev's case of loops, one has a canonical choice of $\Delta$. Namely, in $T(P)$, we can define the coproduct by (3.2.6), (3.2.7) except replacing $l_{H}$ in (3.2.6) by an ordered tensor product, choosing the left-to-right order of components induced by the original ordering on the path of the left endpoints of each component. As in [Tur05], it is easy to check that this gives a Hopf algebra and that a suitable version of the homomorphism $\eta$ maps this Hopf algebra to Foissy's noncommutative algebra of labeled rooted trees [Foi02] (the rooted trees must have labeled edges, or equivalently, labeled vertices in order to capture the left-to-right order that we obtain in paths). So more "noncommutative" or "ordering" information is included in paths or loops than in rooted trees without labels.

On the other hand, one can obtain a generalization of Proposition 3.5.2 if one imposes the additional condition that $\Delta_{n}(V) \subset \operatorname{Sym}^{n} V \otimes V \subset T^{n}(V) \otimes V$. In this case, there is not really anything new to check since coassociativity is proved in $\operatorname{Sym}(V) \subset T(V)$ and extends to all of $T(V)$ using the bialgebra condition (since we define $\Delta(f g):=\Delta(f) \Delta(g))$. This might be the dual version of the noncommutative structure hinted at in Remark 2.14 of [OG05]. However, this is not the way to construct the noncommutative Hopf algebras described above, since they use extra structure (the ordering in paths, or in the case of trees, the labeling on edges and/or vertices) and their coproducts do not map $V$ to $(\operatorname{Sym} V \otimes V) \oplus(1 \otimes V)$.

\section{Postponed proofs}

4.1. Proof of Proposition 3.2.8. We only need to check coassociativity. Then the bialgebra property essentially follows from the definition. Coassociativity is easily verified by an explicit formula as follows.

Definition 4.1.1. For any path $X=a_{1} \ldots a_{n} \in P$, any cut $H$ of $X$ and any vertex $v$ of $L_{X}$ (so $v \in \mathbb{Z}+\frac{1}{2}$ and $\frac{1}{2} \leq v \leq n+\frac{1}{2}$ ), define the $\operatorname{order} \operatorname{ord}(H, v)$ to be the minimum number of chords (the edges connecting $i_{\ell}$ and $j_{\ell}$ if $H=\left\{\left(i_{\ell}, j_{\ell}\right)\right\}_{1 \leq \ell \leq m}$ ) 
of the chord diagram $C_{X, H}$ that must be crossed by any path from $v$ to the root which intersects the chord diagram $C_{X, H}$ only transversely. If $v$ is the root, we define $\operatorname{ord}(H, \mathrm{rt}):=0$.

Definition 4.1.2. For any path $X$ and cut $H$, let the $\operatorname{order}$ of $H$, ord $(H)$, be defined to be the maximum of all ord $(H, v)$ for vertices $v$ of $L_{X}$.

We note that a nonempty cut is simple iff its order is one.

Definition 4.1.3. For two disjoint cuts $H_{1}, H_{2}$ of a path $X=a_{1} \ldots a_{n}$, we say that $H_{1} \prec H_{2}$ if from all points there is a path, intersecting $H_{1}$ and $H_{2}$ transversally, to the root that does not intersect chords from $H_{1}$ after those from $H_{2}$. In other words, the chords from $H_{2}$ are not separated from the root by any chords from $H_{1}$.

In particular, $\emptyset \prec H$ and $H \prec \emptyset$. Note that $\prec$ satisfies the transitivity property.

We now may give the formula

$$
(\Delta \otimes 1) \Delta(X)=\Delta(X) \otimes 1+\sum_{1 \leq \operatorname{ord}(H) \leq 2} \varepsilon_{H} \sum_{\substack{\text { simple cuts } H_{1}, H_{2} \\ \text { such that } H=H_{1} \\ H_{1}<H_{2}}} l_{H_{2}} \otimes l_{H_{2}, H_{1}} \otimes r_{H_{2}},
$$

where $l_{H_{2}, H_{1}}$ is the product of all paths along $L_{X}$ cut from the chord diagram for $H$ which lie between a chord from $H_{1}$ and a chord from $H_{2}$. The same formula holds for $(1 \otimes \Delta) \Delta(X)$, which implies the result.

4.2. Proof of Theorems 3.3.16, 3.4.10. We prove Theorem 3.4 .10 as well as injectivity of the composition $D_{*} \circ S_{*}$, which implies Theorem 3.3.16.

First, we show that $S_{*}$ is a homomorphism of pre-Lie or Lie coalgebras. For this, we note that a cut $H$ together with a specified chord $c \in H$ is the same information as a specified chord $c$ together with two cuts $H_{1}, H_{2}$, one on each side of $c$, under the correspondence $H=H_{1} \sqcup H_{2} \sqcup\{c\}$. The datum $(H, c)$ corresponds to a summand in the expression for $\delta_{*} \circ S_{*}$ of a given (cyclic) path, while the datum $\left(c, H_{1}, H_{2}\right)$ corresponds to a summand in the expression for $\left(S_{*} \otimes S_{*}\right) \circ \delta_{*}$. It is then easy to see that the two summands are identical.

Next, we show that $S_{\mathrm{rt}}$ extends to a homomorphism of Hopf algebras (3.4.3). We only need to check that $S_{\mathrm{rt}}$ sends the coproduct on Sym $P$ to the coproduct on Sym $C h_{\mathrm{rt}}$. For this, we extend the observation of the previous paragraph: the datum $\left(H, H_{1}\right)$ of a cut $H$ and a simple subcut $H_{1}$ (corresponding to a summand of $\left.\Delta \circ S_{\mathrm{rt}}(X)\right)$ yields the same information as the datum $\left(H_{1},\left\{H_{c}^{\prime}\right\}_{c \in H_{1}}, H_{0}^{\prime}\right)$, where $H_{1}$ is a simple cut, and $\left\{H_{c}^{\prime}, H_{0}\right\}_{c \in H_{1}}$ is a collection of cuts on the connected components $\left\{X_{H_{1}, c}\right\}_{c \in H_{1}} \sqcup\left\{X_{H_{1}, 0}\right\}$ which result from cutting $X$ along $H_{1}$ (corresponding to a summand of $\left.\left(S_{\mathrm{rt}} \otimes S_{\mathrm{rt}}\right) \circ \Delta(X)\right)$. This correspondence is given by 
$H=H_{1} \sqcup \bigsqcup_{c \in H_{1}} H_{c}^{\prime} \sqcup H_{0}^{\prime}$. Corresponding data produce identical summands of $\Delta \circ S_{\mathrm{rt}}(X)$ and $\left(S_{\mathrm{rt}} \otimes S_{\mathrm{rt}}\right) \circ \Delta$ given by

$$
\varepsilon_{H_{1}} l_{H_{1}}(X)_{\left\{H_{c}^{\prime}\right\}_{c \in H_{1}}} \otimes r_{H_{1}}(X)_{H_{0}^{\prime}},
$$

where the subscripts of $H_{0}^{\prime}, H_{c}^{\prime}$ indicate which chord diagrams to attach to the corresponding paths in the above monomial of paths.

Next we show that $D_{*}$ is a homomorphism. First we tackle the pre-Lie, Lie cases. For any chord diagram $C_{X, H}$, we need to show that $\left(D_{*} \otimes D_{*}\right) \delta_{*}\left(C_{X, H}\right)=$ $\delta_{*} \circ D_{*}\left(C_{X, H}\right)$. First, note that a choice of chord of a chord diagram is the same as a choice of edge of the dual tree. It is easy to see that the same tree-structure is obtained by either cutting along this chord and then applying $D_{*}$ (dualizing) or applying $D_{*}$ first and then removing the corresponding edge. It remains to show that, for any chord $c \in H$, we have

$$
\varepsilon_{H \backslash c} \varepsilon_{c}=\varepsilon_{H},
$$

which follows from the definition (and was first noticed in [Tur05]).

In the Hopf algebra setting (3.4.2), showing $D_{\mathrm{rt}}$ is a homomorphism amounts to showing, for any chord diagram $C_{X, H}$, that $\left(D_{\mathrm{rt}} \otimes D_{\mathrm{rt}}\right) \Delta\left(C_{X, H}\right)=\Delta\left(D_{\mathrm{rt}}\left(C_{X, H}\right)\right)$. First, we note that a simple subcut of a chord diagram is the same as a simple cut of the dual tree. As before, it remains to show that the signs work out correctly, that is, if $H_{1}$ is a simple subcut of $H$, then

$$
\varepsilon_{H_{1}} \varepsilon_{H \backslash H_{1}}=\varepsilon_{H}
$$

This identity, noticed in [Tur05], is obvious from the definition.

Finally, we show that $D_{*} \circ S_{*}$ is injective. This follows because, for any (cyclic) path $X$, the trivial chord diagram yields a summand of $D_{*} \circ S_{*}(X)$ which is the trivial tree (a point) whose rooted or oriented tree-structure at that point includes $X$ itself. All other summands are trees with $\geq 1$ edges. So if we compose $D_{*} \circ S_{*}$ with the projection to the space spanned by the trivial tree with arbitrary structure, we easily obtain the (cyclic) path $X$.

Note that if the tree-structure is forgotten and we take the map to the [CK98] algebra of trees itself, the composition is not injective; e.g., any path without any pair of edges of the form $e, e^{*}$ for $e \in Q$ must map to the trivial tree.

\section{References}

[AMR96] J. E. Andersen, J. Mattes, and N. Reshetikhin, The Poisson structure on the moduli space of flat connections and chord diagrams. Topology 35 (1996), 1069-1083. Zbl 0857.58009 MR 1404925

[BLB02] R. Bocklandt and L. Le Bruyn, Necklace Lie algebras and noncommutative symplectic geometry. Math. Z. 240 (2002), 141-167. Zbl 1113.16019 MR 1906711 
[CK98] A. Connes and D. Kreimer, Hopf algebras, renormalization and noncommutative geometry. Comm. Math. Phys. 199 (1998), 203-242. Zbl 0932.16038 MR 1660199

[CL01] F. Chapoton and M. Livernet, Pre-Lie algebras and the rooted trees operad. Internat. Math. Res. Notices 20001 (2001), 395-408. Zbl 1053.17001 MR 1827084

[Foi02] L. Foissy, Les algèbres de Hopf des arbres enracinés décorés, II. Bull. Sci. Math. 126 (2002), 249-288. Zbl 1013.16027 MR 1909461

[Ger63] M. Gerstenhaber, The cohomology structure of an associative ring. Ann. of Math. (2) 78 (1963), 267-288. Zbl 0131.27302 MR 0161898

[Gin01] V. Ginzburg, Non-commutative symplectic geometry, quiver varieties, and operads. Math. Res. Lett. 8 (2001), 377-400. Zbl 1113.17306 MR 1839485

[Gol84] W. M. Goldman, The symplectic nature of fundamental groups of surfaces. Adv. in Math. 54 (1984), 200-225. Zbl 0574.32032 MR 762512

[Kre98] D. Kreimer, On the Hopf algebra structure of perturbative quantum field theories. Adv. Theor. Math. Phys. 2 (1998), 303-334. Zbl 1041.81087 MR 1633004

[OG05] J.-M. Oudom and D. Guin, Sur l'algèbre enveloppante d'une algèbre pré-Lie. C. $R$. Math. Acad. Sci. Paris 340 (2005), 331-336. Zbl 02153797 MR 2127105; see also: On the Lie envelopping algebra of a pre-Lie algebra, arXiv:math/0404457

[Sch05] T. Schedler, A Hopf algebra quantizing a necklace Lie algebra canonically associated to a quiver. Internat. Math. Res. Notices (2005), 725-760. Zbl 1079.16028 MR 2146606

[Sch07] T. Schedler, Hochschild homology of preprojective algebras over the integers. Preprint 2007; arXiv:0704.3278

[Tur91] V. G. Turaev, Skein quantization of Poisson algebras of loops on surfaces. Ann. Sci. École Norm. Sup. (4) 24 (1991), 635-704. Zbl 0758.57011 MR 1142906

[Tur05] V. Turaev, Loops on surfaces, Feynman diagrams, and trees. J. Geom. Phys. 53 (2005), 461-482. Zbl 1094.57022 MR 2125403

[Vin63] È. B. Vinberg, The theory of homogeneous convex cones. Trudy Moskov. Mat. Obšč. 12 (1963), 303-358; English transl. Trans. Moscow Math. Soc. 12 (1963), 340-403. Zbl 0138.43301 MR 0158414

Received March 8, 2007

W.L. Gan, Department of Mathematics, University of California, Riverside, CA 92521, U.S.A.

E-mail: wlgan@math.ucr.edu

T. Schedler, Department of Mathematics, University of Chicago, 5734 S. University Ave, Chicago, IL 60637, U.S.A.

E-mail: trasched@math.uchicago.edu 